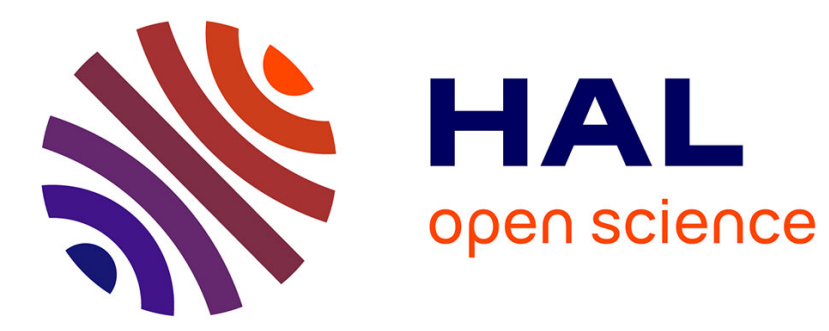

\title{
New evidence of GOE statistics for compound nuclear resonances
}

\author{
M. Lombardi, O. Bohigas, T. H. Seligman
}

\section{To cite this version:}

M. Lombardi, O. Bohigas, T. H. Seligman. New evidence of GOE statistics for compound nuclear resonances. Physics Letters B, 1994, 324, pp.263-266. 10.1016/0370-2693(94)90191-0 hal-00974248

\section{HAL Id: hal-00974248 \\ https://hal.science/hal-00974248}

Submitted on 5 Apr 2014

HAL is a multi-disciplinary open access archive for the deposit and dissemination of scientific research documents, whether they are published or not. The documents may come from teaching and research institutions in France or abroad, or from public or private research centers.
L'archive ouverte pluridisciplinaire HAL, est destinée au dépôt et à la diffusion de documents scientifiques de niveau recherche, publiés ou non, émanant des établissements d'enseignement et de recherche français ou étrangers, des laboratoires publics ou privés. 


\title{
New evidence of GOE statistics for compound nuclear resonances
}

\author{
M. Lombardi \\ Laboratoire de Spectrométrie Physique. Université Joseph Fourier de Grenoble, BP 87, 38402 Saint Martin d'Hères Cedex, \\ France
}

\author{
O. Bohigas ${ }^{1}$ and T.H. Seligman ${ }^{2}$ \\ Wissenschaftskolleg zu Berlin, Berlin, Germany
}

\begin{abstract}
New statistical measures are applied to the previously compiled nuclear data ensemble in order to further test energy level fluctuations as well as the absence of correlations between levels and intensities. Data are found to be consistent with the predictions of the Wigner-Dyson random matrix model.
\end{abstract}

The nuclear data ensemble has been compiled and analysed in reference [1]. There the statistical fluctuations of level sequences, mainly coming from compound nuclear resonances, have been found in excellent agreement with the GOE (Gaussian orthogonal ensemble) prediction [2]. Further analyses including amplitudes [3], as well as higher than two-point correlations [4] confirmed these findings. Yet recently additional more stringent tests were proposed concerning both level statistics [5] and correlations between levels and intensities [6]. In the present article we analyse the nuclear data ensemble according to these new very sensitive tests.

First consider the following result given by Dyson and Mehta [7]: If we have a GOE level sequence and if we omit every other level, we obtain two sequences each of which follows GSE (Gaussian symplectic ensemble) statistics. It is therefore possible to perform this operation on any spectrum and proceed to make statistical analysis on the two resulting spectra. This in itself may not seem very excit-

\footnotetext{
1 Permanent address: Division de Physique Théorique, Institut de Physique Nucléaire, 91406-Orsay Cedex, France. 2 Permanent address: Laboratorio de Cuernavaca, Instituto de Física, University of Mexico (UNAM), Cuernavaca, Mexico.
}

ing, despite of the fact that more complicated combination of the n-point functions enter even if we only look at the two-point function of the new sequences. Yet there is more to this if we consider the square of the Fourier transforms $|C(t)|^{2}$ of the spectra. This is so because the Fourier transform in the GSE case does not only have the characteristic "correlation hole" [8] at small frequencies, which is due to the long range stiffness of the spectrum, but in addition a very marked feature namely a singularity at frequency 1 [2]. This singularity corresponds to oscillations that in principle can be seen in the two-level cluster function $Y_{2}(E)$ or the number variance $\Sigma^{2}(L)$, but are most spectacular in the Fourier transform or the two-body form factor $b(t)$ related to the Fourier transform by $|b(t)-1|^{2}=|C(t)|^{2}$. Notice that by taking the absolute value of $C(t)$, all information on higher than two-point functions disappears.

Let us recall [2] that $b(t)$ is an even function of $t$, and that for the GOE it reads as

$$
b(t)=\begin{array}{lc}
1-2 t+t \ln (1+2 t) & 0<t<1 \\
-1+t \ln ((2 t+1) /(2 t-1)) & t>1
\end{array}
$$

while for the GSE we have 


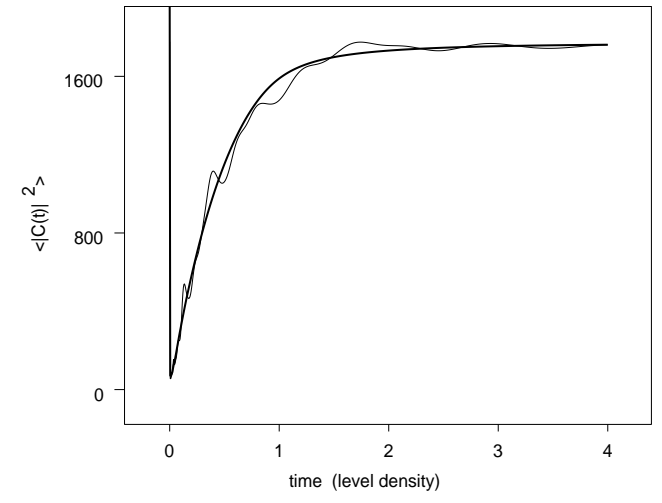

Fig. 1. Smoothed Fourier transform of the Nuclear Data Ensemble. Stick spectra displaying GOE statistics and full correlation hole. Spectra have been unfolded with a second order polynomial. Gaussian smoothing with width increasing with time (equal to $10 \%$ of time). The theoretical curve (heavy line) has been smoothed the same way and contains no adjustable parameter.

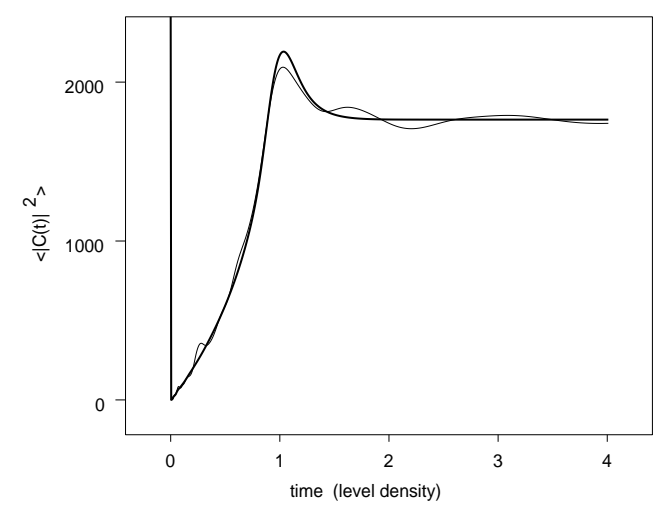

Fig. 2. Smoothed Fourier transform of the Nuclear Data Ensemble omitting every other level and averaged over both sequences. Stick spectra displaying GSE statistics. Spectra have been unfolded with a second order polynomial. Gaussian smoothing with constant width (0.1 FWHM in level density units). The theoretical curve (heavy line) has been smoothed the same way and contains no adjustable parameter.

$b(t)=\begin{array}{lc}1-\frac{1}{2} t+\frac{1}{4} t \ln (|1-t|) & 0<t<2 \\ 0 & t>2 .\end{array}$

At $t= \pm 1, b(t)$ and its first two derivatives are continuous for the GOE, whereas for the GSE there is a singularity. This is connected with the fact that the oscillating term in $Y_{2}(E)$ is, for large $E$, of the order $E^{-4}$ for a GOE and of the order $E^{-1}$ for a GSE.

For the purpose of reference Fig. 1 shows [8] the square of the Fourier transform of all energy spectra compiled in the nuclear data ensemble [1]. Fig. 2 shows the square of the Fourier transform of the spectrum obtained by omitting every other

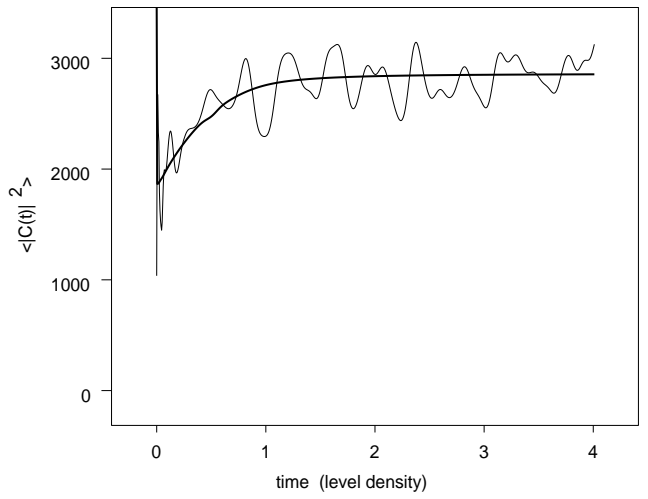

Fig. 3. Smoothed Fourier transform of the Nuclear Data Ensemble. Neutron width weighted spectra displaying reduced correlation hole. Spectra have been unfolded with a second order polynomial. Average width of each spectrum has been normalized to one. Gaussian smoothing with width increasing with time (equal to $10 \%$ of time). The theoretical curve (heavy line) has been smoothed the same way and contains no adjustable parameter. The Nuclear Data Ensemble shows no indication of intensity spacing correlations under this test.

level and averaged over both sequences. We find excellent agreement between the data and the GSE prediction if we disregard the speckle pattern in the Fourier transform of the data.

For a long time the possible correlations between intensities and energies have been considered only by investigating their correlation coefficient. In particular, it has been found [3] that its value, when the nuclear data ensemble is analysed, is consistent with zero, as the random matrix model predicts. Recently a marked correlation in the case of Rydberg molecules was found [6] , to which the test of Ref. [3] is not sensitive. In the same reference it was suggested to consider the Fourier transform of an intensity spectrum defined by $\sum I_{i} \delta\left(E-E_{i}\right)$ in addition to the conventional one of the energy spec$\operatorname{trum} \sum \delta\left(E-E_{i}\right)$. Here $I_{i}$ is the intensity that can be the square of an amplitude or the partial width of a resonance. For the GOE, amplitudes are independently distributed according to a Gaussian. This leads to a Porter-Thomas distribution for the intensities [2] and to the multiplication of $b(t)$ by a factor of $1 / 3$ in the formula relating $b(t)$ to $|C(t)|^{2}[6,8]$. We can study the Fourier transform of the intensity spectra of the nuclear data ensemble for those cases where partial width are available (approx. 1200 levels). The results as obtained in Ref. [6] are shown in Fig. 3 and compared with the exact result. We can see the reduction of the correlation hole as expected.

The main advantage of the use of this procedure is that it is not sensitive to a lack of knowledge about 


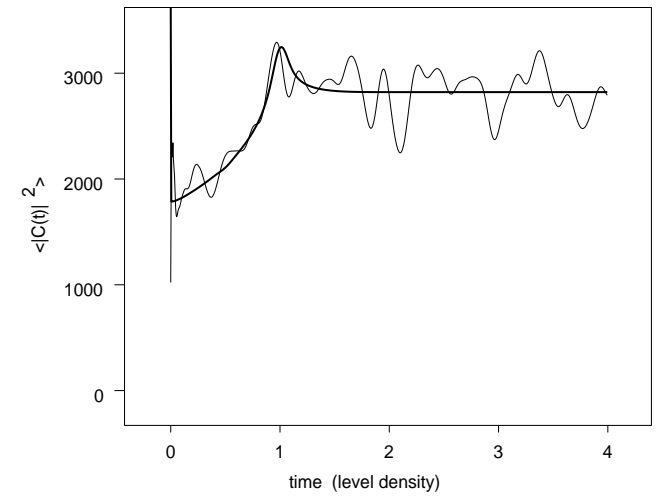

Fig. 4. Smoothed Fourier transform of the Nuclear Data Ensemble omitting every other level and averaged over both sequences. Neutron width weighted spectra displaying GSE statistics curve reduced by intensity effect. Spectra have been unfolded with a second order polynomial. Gaussian smoothing with constant width (0.1 FWHM in level density units). The theoretical curve (heavy line) has been smoothed the same way and contains no adjustable parameter. The $\mathrm{Nu}-$ clear Data Ensemble shows no more indications of intensity spacing correlations under this test.

states with small intensities as their effect on the Fourier transform is small. The same holds for lack of experimental resolution of very close states.

The result for nuclei, shown in Fig. 3, must be seen in contrast to molecular situations were the short time behaviour of the Fourier transform depends sensitively on the channel due to correlations [6]. This was shown to be strictly due to energyamplitude correlations as a random permutation of the intensities on the spectrum eliminated the deviations. Note that the entire effect can only be seen for the shortest times as $b(t)$ rapidly approaches zero for longer times. In fact it was shown that, when the semi-classical approximation is valid (which was the case in Rydberg molecules but is extremely dubious in the case of nuclei), this energy-amplitude correlation effect appears for times shorter than the time needed in classical mechanics for the system to mix on a scale given by the wavelength, so that it has a very interesting dynamical meaning.

To have an other test we may use the above procedure of splitting the spectra into two sequences for intensity spectra. A GOE intensity spectrum analysed in this way will yield level correlations typical of a GSE, but the amplitudes will continue to be those of a GOE. Then random matrix theory predicts the same factor $1 / 3$ weighting $b(t)$ in the Fourier transform $|C(t)|^{2}$ which we know from the GOE itself.

The results of such an analysis for the nuclear data ensemble are shown in Fig. 4. Again we see that the nuclear spectra are quite compatible with the predicted independence of intensities and energies. Note though that in this case we must know all energy levels as the splitting of the spectrum cannot be performed without this knowledge.

Summarising we can say that new very sensitive tests for the analysis of spectral data have been applied to the nuclear data ensemble and that no disagreement with the GOE hypothesis could be detected. These findings are in line with the result of a recent controversy about the validity of statistical assumptions for neutron resonances, which also confirmed the statistical properties $[9,10]$.

\section{References}

[1] R.U. Haq, A. Pandey and O. Bohigas, Phys Rev. Lett. 48 (1982) 1086.

[2] C.E. Porter, Statistical Theory of Spectral Fluctuations, (Academic Press, New York, 1965); M.L. Mehta, Random Matrices and the Statistical Theory of Energy Levels, (Academic Press, New York 1990).

[3] O. Bohigas, R.U. Haq and A. Pandey, in: Nuclear Data for Science and Technology, ed. K.H.Boeckhoff (Reidel, Dortrecht, 1983) p 809.

[4] O. Bohigas, R.U. Haq and A. Pandey, Phys Rev. Lett. 54 (1985) 1645

[5] O. Bohigas, in: Chaos and Quantum Physics, eds. M.J. Giannoni, A. Voros and J. Zinn-Justin, (Elsevier, Amsterdam,1991).

[6] M. Lombardi and T.H. Seligman, Phys. Rev. A 47 (1993) 3571.

[7] M.L. Mehta and F.J. Dyson, J. Math. Phys. 4 (1963) 713.

[8] L. Leviandier, M. Lombardi, R. Jost and J.P. Pique, Phys. Rev. Lett. 56 (1986) 2449.

[9] J. Reckstadt, T.S. Tveter and M. Guttormson, Phys. Rev. Lett. 65 (1990) 2122.

[10] B.R. Barrett et al., Phys. Rev. C 45 (1992) R1417. 